\title{
The Potential Use of the Fetal Observable Movement System in Clinical Practice
}

\author{
${ }^{1}$ Nadja Reissland, ${ }^{2}$ Joe M Austen, ${ }^{3}$ Uiko Hanaoka, ${ }^{4}$ Mohamed Ahmed Mostafa AboEllail \\ ${ }^{5}$ Rina Uematsu, ${ }^{6}$ Toshiyuki Hata
}

\begin{abstract}
This paper summarizes the potential use of a fine-grained fetal facial scoring system. The use of this coding system is discussed in relation to maternal health as well as effects on fetal development, including smoking in pregnancy and maternal stress. It is argued that the proposed fine-grained movement analysis might lend itself to support an increase health behaviors. Furthermore, we suggest that the fine-grained movement coding, although labor intensive, might detect subtle but important differences in fetal development, which are missed by more general observations. Finally, advantages and disadvantages of this system are discussed.
\end{abstract}

Keywords: Prenatal diagnostic, Prenatal movement, Three/ Four-dimensional ultrasound.

How to cite this article: Reissland N, Austen JM, Hanaoka U, AboEllail MAM, Uematsu R, Hata T. The Potential Use of the Fetal Observable Movement System in Clinical Practice. Donald School J Ultrasound Obstet Gynecol 2015;9(4):426-433.

\section{Source of support: Nil}

Conflict of interest: None

\section{INTRODUCTION}

Ultrasound examinations have become a routine part of maternal prenatal care, but researchers increasingly accept the idea that not only two-dimensional (2D) sonographic examinations but also the more sophisticated three- (3D)/four-dimensional (4D) ultrasound technique could be used as a diagnostic tool in routine practice. This is due to the greater number of sonographers proficient in 3D/4D ultrasound scanning as well as technical improvements of the tools used for ultrasound imaging. This could have a profound impact on not only clinical practice in terms of identifying fetal anomalies but also in terms of counseling parents about these anomalies. Another advantage of the 3D/4D images could be to

\footnotetext{
${ }^{1,3}$ Associate Professor, ${ }^{2}$ Postdoctoral Fellow, ${ }^{4}$ Research Fellow ${ }^{5}$ Sonographer, ${ }^{6}$ Professor and Chairman

1,2Department of Psychology, Durham University, United Kingdom

${ }^{3-6}$ Department of Perinatology and Gynecology, Kagawa University Graduate School of Medicine, Kagawa, Japan
}

Corresponding Author: Nadja Reissland, Associate Professor Department of Psychology, Durham University, Science Site, South Road, Durham DH1 3LE, United Kingdom, e-mail:n.n.reissland@ durham.ac.uk potentially increase parental health behaviors when seeing their fetus as a 'baby' in need of good nutrition and care.

In terms of advantages in clinical practice, a study by Picone et al, ${ }_{1}^{1}$ compared the level of detail seen in an ultrasound examination the week before a termination of pregnancy (TOP) and the results of the autopsy conducted after TOP. They did not report the age of the termination apart from stating that the study examined terminations after 21 weeks of pregnancy. According to their results, Picone et $\mathrm{al}^{1}$ claimed that ultrasound was suboptimal when identifying anomalies when compared to autopsy, with the exception of malformations of the fetal face and spine. It remains unclear whether the detection of various anomalies by ultrasound might be dependent on the age of the fetus (and potentially could show improved results for older vs younger fetuses) or alternatively might be dependent on the quality of the ultrasound image obtained. Their study also ignores the additional potential of ultrasound studies, which can detect not only the presence or absence of fetal movements, but also their quality. Although Picone et $\mathrm{al}^{1}$ did not find ultrasound scans to be as accurate as the examination by autopsy, the advances in the technique might ultimately make the reliance on autopsies less essential. Furthermore, the advances in 4D ultrasound technology might potentially replace the need for autopsies all together.

A recent overview of 25 years of ultrasound ${ }^{2}$ in clinical practice argued that $3 \mathrm{D} / 4 \mathrm{D}$ ultrasound can be used not only for diagnostics for example of nuchal transparency and nasal bone measurements in the first trimester but also tomographic analyses of the fetal brain, visualizing vascular anomalies as well as brain malformations. Regarding defects of the fetal face, such as cleft lip, as well as the spine or extremities $3 \mathrm{D} / 4 \mathrm{D}$ ultrasound is increasingly used in diagnosis. Additionally, it helps with counseling parents not only of fetal malformations but also to reassure them that the fetus is developing normally. ${ }^{3}$ The surface mode rendering of 3D/4D is especially relevant in this context. However, visualization is not enough in order to make the technique useful in clinical practice rather the images need to be coded and compared across laboratories. Below is a description of potential uses of 3D/4D ultrasound using a specific coding system. 


\section{Fetal Observable Movement System}

The fetal observable movement system (FOMS) ${ }^{4}$ is based on coding each anatomically different movement individually and enables the standardized investigation of fetal facial movements. There are a number of anatomically based systems that have been developed mainly for adult facial expressions and adapted for children and infants (e.g. Facial Action Coding System, ${ }^{5}$ the Album des expressions du visage, ${ }^{6}$ the Maximally Discriminative Facial Movement Coding System MAX). ${ }^{7}$ These systems have provided a standardized tool for investigating human facial expression.

In contrast to these standardized tools used postnatally, coding systems for fetal facial expressions that attempt to chart the development of facial movement rely on the subjective interpretation of facial muscle movements, and thereby do not allow a comparison across studies. ${ }^{8-10}$ For example, Piontelli ${ }^{10}$ labeled one fetal facial expression as a 'smile', suggesting that smiling is 'anatomically the simplest of all expressions' and has been recorded from around 15 to 16 weeks gestation. Such an interpretation is too general when using the FOMS, where an expression that could be interpreted as a smile might be one of three movements coded in FOMS: a lip pull, a mouth stretch or a lip stretch. Hence, to label a fetal facial movement as smile could be misleading and not be comparable across studies. Cohn and Ekman ${ }^{11}$ concur with this interpretation by pointing out that although a smile is principally based on one single zygomatic major muscle, it typically involves two or three other muscles as well. Moreover, since smiles vary from individual to individual, they can involve a number of different constellations of muscle movements. In contrast to general movement labeling systems, in anatomically based coding systems coding is objective and can reach, through training, a high degree of reliability between coders.

Although a great deal of research into the human and nonhuman face has been undertaken, little is known about the action of the human face in utero. It has been suggested that fetal movements in general and fetal facial movements in particular are 'spontaneous expressions of an inherently active nervous system ${ }^{\prime 2}$ and provide an insight into the development of the nervous system, both normal and abnormal. Such insight can be used in a variety of ways, including charting the development of fetal facial movements that coalesce to produce an 'expression'; moreover, they can also support research into the development of the fetal nervous system. In the context of clinical research, using fetal facial movements as markers could be applied during routine scanning. When fetal movements are labeled with emotionally charged terminology (e.g. 'smile') or there is a failure to define movements precisely enough to avoid observations being subjective and investigations hard to replicate, such systems might outweigh the advantages of a simplified coding system. In contrast, the FOMS standardizes the investigation of fetal facial movements, in a similar way to that in which Facial Action Coding System has enabled objective and reliable research on adult facial expression.

\section{An Evolving Coding System}

The fetal coding system FOMS has evolved over time. Originally, we identified 19 facial movements that occur independently of one another and are visible using $4 \mathrm{D}$ ultrasound scans of the fetus. ${ }^{13}$ The current system includes not only these 19 facial movements but also touch behaviors. Although this system has only been used to investigate the movement patterns of fetuses aged between 23 and 37 weeks gestation, given research indicating that fetal facial expressions develop from around 15 to 16 weeks ${ }^{10}$ and that innervations of these muscles start from around 8 weeks gestation, ${ }^{14}$ it is likely that FOMS could readily be applied to fetuses of a younger gestational age.

Currently there are some systems, which use the quality of movements, such as floppy movements, to identify potential developmental problems. However, not only the quality of movements but also the dynamics of the movements might be of interest in clinical practice. Using the FOMS to code the dynamics of one specific movement (mouth stretch), we reported that this same movement could be used to distinguish between simple mouth movement and yawning ${ }^{15}$ (Fig. 1).

Some researchers suggest that fetal yawn behavior might reflect activity-dependent brain maturation ${ }^{16}$ and therefore yawning could indicate healthy brain development. In FOMS we can distinguish between 'yawning' and a non-yawn 'mouth stretch' whereby a
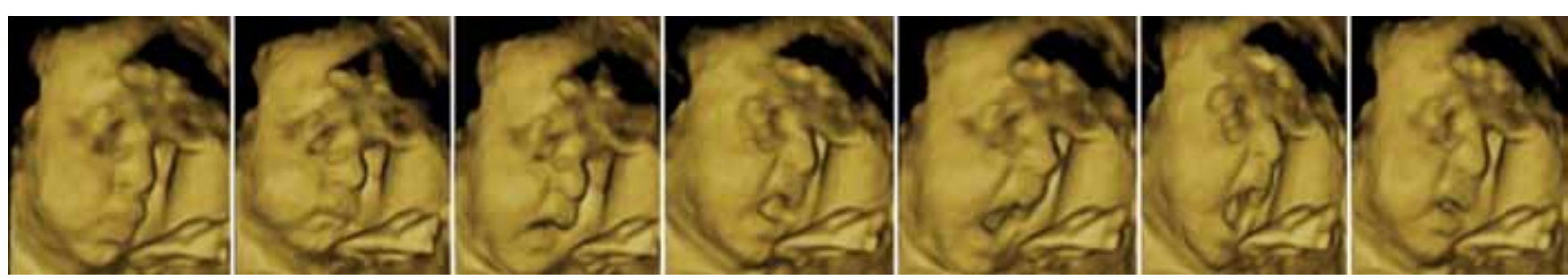

Fig. 1: Thirty-two-week-old fetus yawning 
mouth stretch is defined as the mandible being lowered, changing the shape of the mouth opening from oval with the long axis in the horizontal plane to the long axis of mouth opening in the vertical plane. The dynamics of the movement is essential in distinguishing between yawns and simple mouth stretch. This definition of yawning adopted in this study was based on Petrikovski et $\mathrm{al}^{17}$ who specified that yawning was a slow opening of the mouth (50-75\% of the cycle) and faster return to the initial position (5-10\% of the cycle). This dynamic of the mouth opening and closing cycle characteristic for yawning could not be seen in the non-yawning mouth stretches, which typically involve mouth opening and closing at similar speeds.

Petrikovsky et al ${ }^{17}$ suggested that yawning could potentially be a clinical indicator of prenatal anemia. Specifically, they pointed out that anemic fetuses showed bursts of yawning activity, which could not be observed in healthy fetuses. They argued that yawning bursts could potentially affect intrathoracic pressure, and thereby increase venous return to the heart. In the case of anemic fetuses, these yawning bursts could be a compensatory mechanism not present in healthy fetuses. In the healthy group of fetuses observed from 24 to 36 weeks gestation using 4D ultrasound the frequency of yawning declined from 24 to 36 weeks gestation. Such decline has also been observed in 31 to 40 weeks old low risk infants. ${ }^{18}$ They argued that the significant decrease in the frequency of yawning that they observed in this population might be associated with the development of the circadian and homeostatic control of sleep and wake cycles, which are controlled by the central nervous system (CNS). Hence, one might argue that a delay in the decline of yawning frequencies might potentially be an indicator of variance to normal CNS maturation.

\section{Using FOMS to identify Developmental Movement Trajectories of Fetuses exposed to Nicotine}

It is well established in the literature that exposure to smoking post-birth is a crucial risk factor for poor
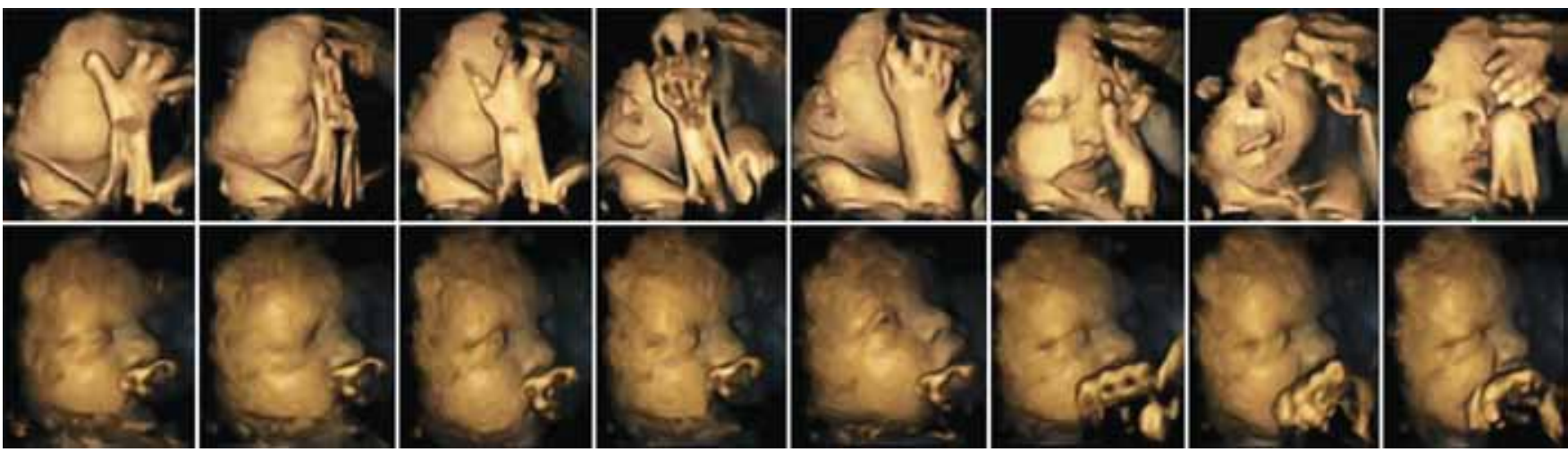

Fig. 2: Comparison of movements between fetuses exposed (top) and not exposed (bottom) to nicotine 
Therefore, by using FOMS we are able to detect fine-grained movement differences that might reflect a delay in fetal CNS maturation as supported by research of Huizink and Mulder. ${ }^{23}$ These differences in CNS maturation are shown in our sample of fetuses exposed to smoking in that they showed a significant delay compared with fetuses of nonsmoking mothers in terms of the rate at which movement behaviors declined with gestational age. Given that post-birth delays in children who were exposed to smoking during their fetal development, such as delays in speech processing, ${ }^{24}$ are well documented, it seems that FOMS might have a role to play in the early detection of delays of development or abnormal developmental trajectories in fetal life.

\section{Maternal Self-reported Stress and Laterality of Fetal Self-touch}

Research has established that in human fetuses cortical lateralization takes place between 15 and 26 weeks gestation. ${ }^{25}$ Maternal stress during pregnancy might influence or disturb the normal process of lateralization. Given that high levels of stress during the prenatal period alter the biochemical equilibrium of the uterus and that research indicates that abnormally elevated prenatal maternal stress is a risk factor for developmental disorders postnatally, ${ }^{26}$ fetal behavior in relation to maternal stress levels is of clinical importance.

Reissland et $\mathrm{al}^{27}$ examined lateralized behaviors by coding self-touch in fetuses using FOMS (Fig. 3). Our results showed that maternal prenatal stress is associated with lateralized self-touch. Fetuses of mothers who reported elevated levels of stress had fetuses that preferentially used their left rather than right hand to touch their face. Moreover, this increase in left-handed touching appeared to be temporally related to the period in which the mother reported stress, with mothers who reported being stressed in the period 2 months before

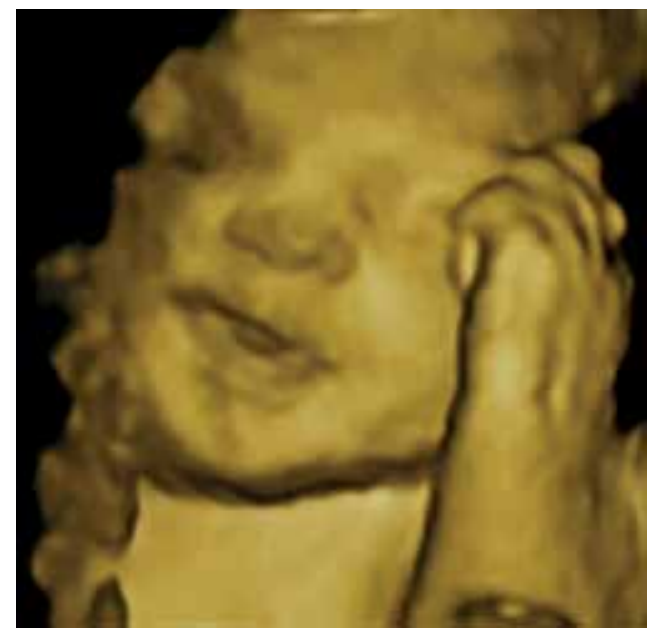

Fig. 3: Fetus of 32 weeks gestational age exhibiting left-handed self-touch the scan, but were not stressed in the month immediately before the scan not having fetuses that showed this left lateralized preferential touch behavior.

These results indicate that current but not historic stress seems to influence fetal lateralized. It is well established that stress during pregnancy has the effect of increasing the levels of cortisol produced by mothers and being transferred to the fetus. This might be a possible mechanism for influencing the development of the fetal brain and consequently producing abnormal laterality post birth. ${ }^{28}$ Using our fine-grained analysis when applying the FOMS coding we established that laterality of self-touch is neither consistent with, nor related to, gestational age of the fetus. Hence, we did not find that increasing maturity of the fetuses resulted in more right handed self-touch as would be expected given that right handedness is considerably more common than left handedness after birth.

Post-birth in human and nonhuman animals the asymmetric development of the brain is well established. However, in fetal research, there is still a debate concerning the prenatal origins of laterality. Although anatomically it has been established that by the second trimester of pregnancy the left cerebral hemisphere is significantly larger than the right in both male and female fetuses, it is not yet clear whether such brain differences translate into lateralized behaviors exhibited by the fetus. In a longitudinal study examining fetal mouth opening from 24 to 36 weeks ${ }^{29}$ in 60 scans we used FOMS to code two types of mouth opening movements-upper lip raiser and mouth stretch. Analysing the lateralized opening (either right or left mouth opening) indicated that lateralized mouth openings increased by $11 \%$ for each week of gestational age. Hence, lateralization of mouth movements may provide another indicator of fetal maturation, and another potential method of observing abnormal fetal development using FOMS in clinical practice.

\section{Using FOMS to Increase Health Behavior}

Using ultrasound and specifically detailed facial movements might have the potential to increase health behaviors. Barnes ${ }^{30}$ argued that currently pregnant women, as a result of the advances in ultrasound technology, may be more likely to see their fetus as a 'person' during pregnancy compared to women not having any experience of ultrasound imaging of their fetuses. Barnes ${ }^{30}$ found that mothers were influenced by ultrasound technology in the way they perceived their unborn child. Having seen their fetus in ultrasound scans women expressed that they saw the fetus as 'a baby' already engaged in human-like activity'. Also the medical community in the last few decades with the increased 
abilities to treat the fetus and the mother separately has established a two-patient model in which the unborn child and the mother can be seen as having conflicting medical needs. For example, smoking e-cigarettes might help improve maternal health by reducing the amounts of toxins the mother consumes, by just taking in pure nicotine, when compared with smoking cigarettes. However, pure nicotine remains in the fetal circulation for much longer than it does in the maternal circulation. Hence, nicotine replacement therapy might benefit maternal health but hinder fetal development, given that nicotine has been shown to affect fetal brain function. ${ }^{31}$

The two-patient model, which is highlighted when visualizing the fetus, might have a positive effect. By showing an accurate image of the fetus and pointing out similarities, in terms of facial expressions and movements, a father's feeling of attachment may be increased, which could ultimately impact on the care given to the child both pre- and post-natally. Hence, 3D/4D ultrasound, especially when used in conjunction with explanations of the images, might increase health behaviors. We might expect the extent to which health behaviors are affected to differs depending on education, experience and culture. For example, Mitchell and Georges ${ }^{32}$ found that mothers undergoing 4D scans in Canada were more likely to perceive their fetus as already having developed a personality compared with Greek mothers who voiced only a few comments (mostly related to the sex of the unborn child). The authors argued that this difference in attitude to the fetus was due to the different discourse employed by sonographers with Greek and Canadian mothers. It is hoped that the use of a standardized system for understanding fetal movement behaviors, such as FOMS, might help establish a common discourse on movements observed, especially of the fetal face, and thereby make the experience more comprehensible to the general population.

\section{Comparison of Kurjak's Antenatal Neurodevelopmental Test (KANET) and FOMS}

The previous article in this issue describes the use of KANET in clinical practice. This coding scheme has been used for some time and very interesting results have been published. ${ }^{33-35}$ The simple codes used in KANET allow its application in a clinical context.

The FOMS coding scheme has not yet been extensively tested. Hence, this report points out potential uses as well as different requirements for use of KANET and FOMS. Specifically the quality of the scans is an essential limiting factor when completing a comprehensive FOMS analysis of fetal scans.

Kurjak's antenatal neurodevelopmental test, in contrast to the fine-grained movement system of FOMS, allows for scans of lesser quality to be coded reliably. This is not the case with FOMS. The advantages of FOMS however are that fine-grained movements might have more power to differentiate between fetuses that might be affected by lesser degrees of illness. For example, in a small pilot study coding low birth weight (LBW; defined as a birth weight of less than $2500 \mathrm{gm}$ ) and normal birth weight (NBW) fetuses in Japan showed no significant differences between the groups when coded using KANET (Graph 1).

Does the FOMS distinguish between NBW and LBW fetuses and is the scoring system more sensitive than KANET? In order to test this in a preliminary study we compared various movements between NBW and LBW fetuses. Only four of the five LBW fetus scans were of good enough quality to code individual behaviors, and therefore we compared their movements with a group of NBW fetuses matched for gestational age and gender. We also included NBW and LBW groups of United Kingdom (UK) fetuses for comparison.

Examining the total number of mouth movements made by UK and Japanese LBW and NBW fetuses, we found that, although not significant, there is an indication that Japan fetuses make more mouth movements than UK fetuses, and that LBW fetuses make more mouth movements than NBW fetuses (Graph 2). We also split the data into specific mouth movements, but found no obvious patterns (and no significant differences).

Testing the total number of touch behaviors made by UK and Japanese LBW and NBW fetuses, we found that, although not quite significant, again there was an indication that Japanese fetuses show more touch behaviors compared with UK fetuses ( $p=0.057)$, and that LBW fetuses touch more frequently compared with NBW

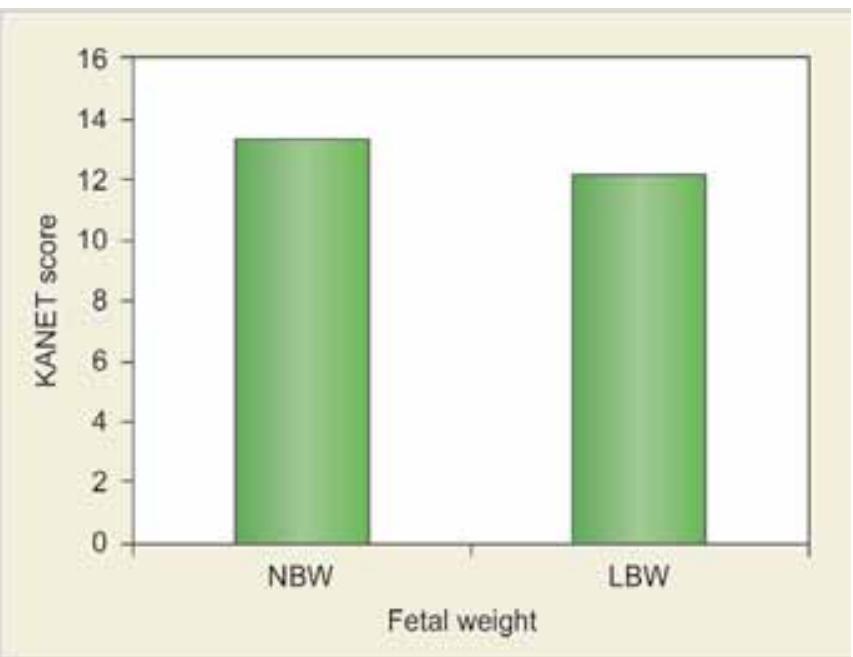

Graph 1: Kurjak's antenatal neurodevelopmental test scores for NBW $(n=45)$ and LBW $(n=5)$ fetuses from Japan. These means are not significantly different: $t(48)=1.0, p=0.32$ 


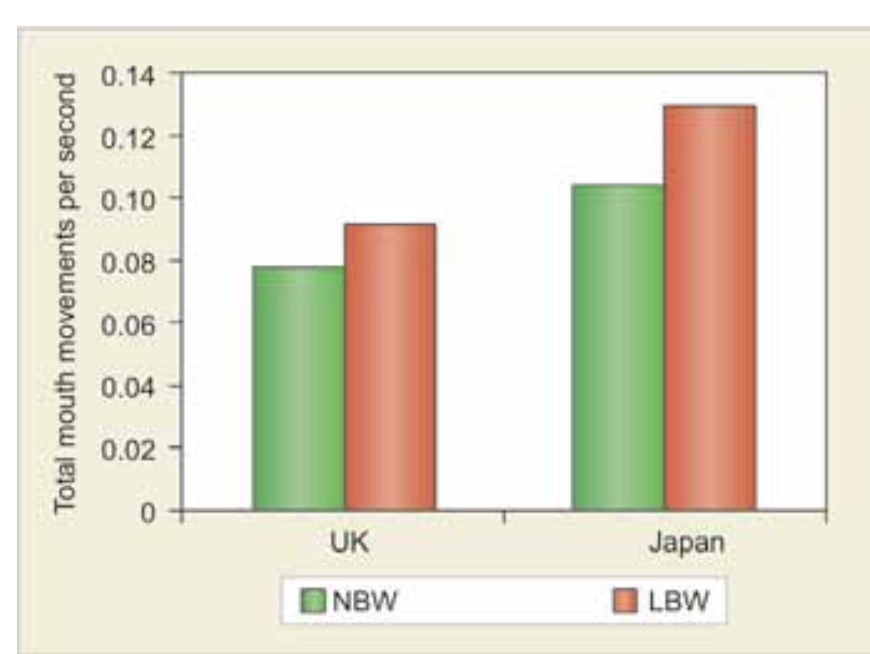

Graph 2: Total mouth movements per second for NBW (UK $\mathrm{n}=$ 3, Japan $n=4)$ and LBW (UK $n=3$, Japan $n=4)$ fetuses from UK and Japan. There are no significant differences among the means

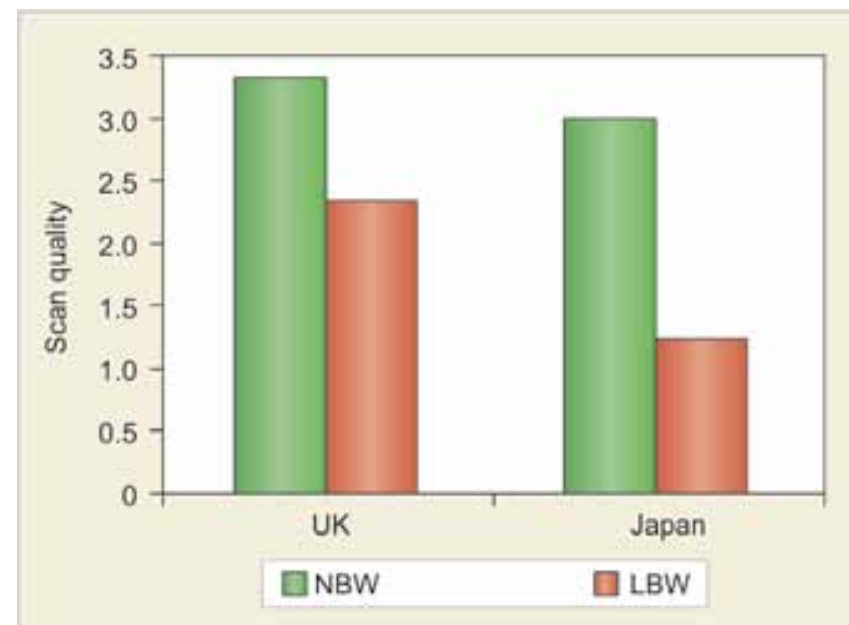

Graph 4: Scan quality for NBW $($ UK $n=3$, Japan $n=4)$ and LBW (UK $n=3$, Japan $n=4$ ) fetuses from UK and Japan

fetuses ( $\mathrm{p}=0.105$; Graph 3). Again, splitting the data by specific type of touch did not yield any obvious patterns (and no significant differences).

From this preliminary study, it can be seen that, although KANET does not appear to distinguish between NBW and LBW fetuses, using a more fine-grained movement coding system such as FOMS does indicate differences between these two groups of fetuses. This would suggest that FOMS is more sensitive to subtle behavioral changes than a system, such as KANET, that addresses only gross motor behavior. As such, FOMS shows high potential for use in a clinical setting for diagnosing abnormal developmental trajectories that other movement-based systems might fail to detect.

One problem when trying to compare the use of KANET with FOMS is that FOMS requires better scan quality since it relies on being able to see fine grained movements. We therefore established a Scan Quality Coding Scheme (Table 1).

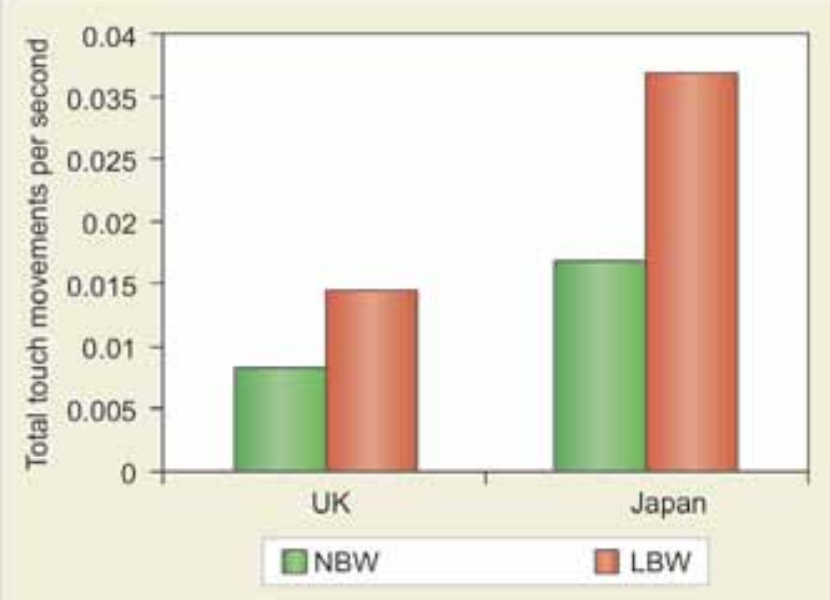

Graph 3: Total touch behaviors per second for NBW (UK $n=3$, Japan $n=4)$ and LBW (UK $n=3$, Japan $n=4)$ fetuses from UK and Japan

Table 1: Scan quality coding scheme

\begin{tabular}{|c|c|c|}
\hline Scan quality & Description & What can be coded? \\
\hline 0. & Uncodable & $\begin{array}{l}\text { Vague outlines of fetal facial } \\
\text { configuration discernible but un- } \\
\text { codable using the FOMS system }\end{array}$ \\
\hline 1. & Poor & $\begin{array}{l}\text { Allows the coding of only very } \\
\text { gross movements }\end{array}$ \\
\hline 2. & Acceptable & $\begin{array}{l}\text { Allows the coding of parts of the } \\
\text { fetal face }\end{array}$ \\
\hline 3. & Good & $\begin{array}{l}\text { A clear view of the fetal face } \\
\text { throughout with few occurrences } \\
\text { of uncodable features of the fetal } \\
\text { face }\end{array}$ \\
\hline 4. & Very good & $\begin{array}{l}\text { A clear view of fetal face with facial } \\
\text { features and movements being } \\
\text { consistently codable }\end{array}$ \\
\hline
\end{tabular}

Whilst the KANET coding system can be applied to scans of quality 1 or higher, for accurate coding using FOMS a minimum scan quality of 2 to 3 is required. In our example, scans, were of a quality that allowed coding using KANET; however, the quality was not good enough especially for the LBW fetuses from the Japanese sample for us to code specific movements in FOMS. Graph 4 demonstrates the low quality of the Japan LBW scans compared with the other groups.

\section{FOMS Use in Clinical Practice}

Throughout this paper we have indicated that FOMS has the potential to be used in clinical practice. More research is essential in order to test the use of this coding system for clinical practice. One drawback is the need for clear images in order to apply our fine-grained movement coding system. However, given the advances in $3 \mathrm{D} / 4 \mathrm{D}$ ultrasound scanning technology, we expect that this will be possible in the near future. Another problem with the use in clinical practice could be that the fine-grained coding necessary for FOMS coding is 
too time consuming. However, we expect that once we can establish which of the behaviors are specifically indicative of conditions, such as the association of maternal self-reported stress and increased left handed touch by fetuses, then sonographers could look for very specific movements, drastically reducing the amount of time required for diagnosis using FOMS. Thus, further advances in ultrasound scanning technology and improved knowledge of which fetal movements are most important to indicate a developmental delay will result in FOMS being a coding system with potential for use in clinical practice.

With the advancement in the technology of 3D/4D ultrasound scanning, we expect that research involving the quantification of fetal facial movements will become more accessible and therefore more commonplace. Given the lack of a fine-grained and reliable coding system based on anatomical movements, which can be observed in $4 \mathrm{D}$ scans, our proposed coding system provides data that can be reported in a format that allows comparison between groups of fetuses observed in various laboratories around the world and in various cultures in more detail than hitherto possible. In addition, there is some indication that fine-grained movement analyses might elucidate fetal conditions, which could be useful for the clinical management of the pregnancy.

\section{REFERENCES}

1. Picone O, Levaillant J-M, Hirt R, Frydman R, Boulvain M, Senat MV. Correlation between referral ultrasound with suspected fetal anomalies and autopsy examination in two prenatal diagnosis centres. Impact of the routine use of 3D/4D scan. Prenat Diagn 2008;28(3):191-196.

2. Merz E. 25 years of 3D Ultrasound in prenatal diagnosis (1989-2014). Ultraschall in Med 2015;36(1):3-8.

3. Merz E. Current 3D/4D ultrasound technology in prenatal diagnosis. Eur Clinics Obstet Gynaecol 2005;1(3):184-193.

4. Reissland N, Francis B, Buttanshaw L. The fetal observable movement system (FOMS). In: Reissland N, Kisilevsky B, editors. Fetal Development Research on Brain and Behavior, Environmental Influences, and Emerging Technologies. Springer US. 2015, in press.

5. Ekman P, Friesen W. Facial action coding system: a technique for the measurement of facial movement. Consulting Psychologists Press, Palo Alto. 1978.

6. Ermiane R, Gergerian E. Atlas of facial expressions (Album des expressions du visage). Paris: La Pensee Universelle 1978.

7. Izard CE. The maximally discriminative facial movement scoring system (Rev. ed.) Unpublished manuscript, University of Delaware, Department of Psychology 1983.

8. Hata T, Kanenishi K, Hanaoka U, Tanaka H. 4D sonographic assessment of fetal neurobehavior. Donald School J Ultrasound Obstet Gynecol 2012;6(2):121-131.

9. Kurjak A, Azumendi G, Vecek N, Kupesic S, Solak M, Varga D, Chervenak F. Fetal and movements and facial expression in normal pregnancy studied by four dimensional sonography. J Perinat Med 2003;31(6):496-508.
10. Piontelli A. Development of normal fetal movements: The first 25 weeks of gestation. NY: Springer. 2010.

11. Cohn JF, Ekman P. Measuring Facial action and automatic facial image analysis. In: Harrigan JA, Rosenthal R, Scherer K, editors. Handbook of nonverbal behavior research methods in the affective sciences. Oxford: OUP 2005;9-64.

12. Hopkins B, Reissland N. Introduction: towards a fetal psychology. Infant Child Dev 2010;19(1):25-126.

13. Reissland N, Francis B, Mason J, Lincoln K. Do facial expressions develop before birth? PLoS One 2011;6(8):e24081.

14. Humprey T. Reflex activity in the oral and facial area of the human fetus. In: JF Bosma, editor. Second symposium on oral sensation and perception. Springfield, IL: Charles C. Thomas. 1967;195-255.

15. Reissland N, Francis B, Mason J. Development of fetal yawn compared with non-yawn mouth openings from 24 to 36 weeks gestation. PLoS ONE 2012;7(11):e50569.

16. Walusinski O. Fetal yawning. Front Neurol Neurosci 2010;28:32-41.

17. Petrikovsky B, Kaplan G, Holsten N. Fetal yawning activity in normal and high-risk fetuses: a preliminary observation. Ultrasound Obstet Gynecol 1999;13(2):127-130.

18. Giganti F, Hayes MJ, Cioni G, Salzarulo P. Yawning frequency and distribution in preterm and near term infants assessed throughout 24-h recordings. Infant Behav Dev 2007;30(4):641-647.

19. Reissland N, Francis B, Kumarendran K, Mason J. Ultrasound observations of subtle movements: a pilot study comparing fetuses of smoking and non-smoking mothers. Acta Paediatr 2015;104(6):596-603.

20. Reissland N, Francis B, Aydin E, Mason, J, Schaal, B. The development of anticipation in the fetus: a longitudinal account of human fetal mouth movements in reaction to and anticipation of touch. Dev Psychobiol 2014;56(5):955-963.

21. Ianniruberto A, Tajani E. Ultrasonographic study of fetal movement. Semin Perinatol 1981;5(2):175-181.

22. DiPietroJA, Hilton SC, Hawkins M, Costigan KA, Pressman EK. Maternal stress and affect influence fetal neurobehavioral development. Dev Psychol 2002;38(5):659-668.

23. Huizink AC, Mulder EJH. Maternal smoking, drinking or cannabis use during pregnancy and neurobehavioral and cognitive functioning in human offspring. Neurosci Biobehav Rev 2006;30(1):24-41.

24. Key PFA, Ferguson M, Molfese DL, Peach K, Lehman C, Molfese VL. Smoking during pregnancy affects speechprocessing ability in newborn infants. Environ Health Perspect 2007;115(4):623-629.

25. Gupta RK, Hasan KM, Trivedi R, Pradhan M, Das V, Parikh NA, Narayana PA. Diffusion tensor imaging of the developing human cerebrum. J Neurosci Res 2005;15(2): 172-178.

26. Van den Bergh BRH, Mulder EJH, Mennes M, Glover V. Antenatal maternal anxiety and stress and the neurobehavioural development of the fetus and child: links and possible mechanisms-a review. Neurosci Biobehav Rev 2005;29(2):237-258.

27. Reissland N, Aydin E, Francis B, Exley K. Laterality of fetal self-touch in relation to maternal stress. Laterality 2015; 20(1):82-94.

28. Glover V, O'Connor TG, Heron J, Golding J, ALSPAC Study team. Antenatal maternal anxiety is linked with atypical handedness in the child. Early Hum 2004;79(2):107-118.

29. Reissland N, Francis B, Aydin E, Mason J, Exley K. Development of prenatal lateralization: evidence from fetal mouth movements. Physiol Behav 2014;131(0):160-163. 
30. Barnes MW. Anticipatory socialization of pregnant women: learning fetal sex and gendered interaction. Sociol Perspect 2015;58(2):187-203.

31. Wickström R. Effects of nicotine during pregnancy: human and experimental evidence. Curr Neuropharmacol 2007;5(3):213-222.

32. Mitchell LM, Georges E. 'Cross-cultural Cyborgs: Greek and Canadian Women's Discourse on Fetal Ultrasound'. Feminist Stud 1997;23(2):373-401.

33. Kurjak A, Miskovic B, Stanojević M, Amiel-Tison C, Ahmed B, Azumendi G, Vasilj O, Andonotopo W, Trudic T, Salihagić-
Kadić A. New scoring system for fetal neurobehavior assessed by three- and four-dimensional sonography. J Perinat Med 2008(1);36:73-81.

34. Stanojević M, Talic A, Miskovic B, Vasilj O, Shaddad AN, Ahmed B, Salihagić-Kadić A, Predojevic M, Vladareanu R, Lebit D. An attempt to standardize Kurjak's antenatal neurodevelopmental test: Osaka consensus statement. Donald School J Ultrasound Obstet Gynecol 2011;5(4):317-329.

35. Hanaoka U, Hata T, Kanenishi K, AboEllail MAM, Uematsu R, Konishi Y, Kusaka T, Noguchi J, Marumo G, Vasilj O, Kurjak A. Does ethnicity have an effect on fetal behavior? A comparison of Asian and Caucasian populations. J Perinat Med 2015. 